\title{
The Decision to Intensify Therapy in Patients with Type 2 Diabetes: Results from an Experiment Using a Clinical Case Vignette
}

\author{
Richard W. Grant, MD, MPH, Karen E. Lutfey, PhD, Eric Gerstenberger, MS, \\ Carol L. Link, PhD, Lisa D. Marceau, MPH, and John B. McKinlay, PhD
}

Purpose: Lack of medication intensification is a widely recognized but poorly understood barrier to effective diabetes care. We used a video case vignette to assess whether patient or physician demographic variables influence the decision to intensify therapy.

Methods: One hundred ninety-two US primary care physicians each viewed one case vignette of an actor portraying a patient who had type 2 diabetes and borderline indications for medication intensification. Case vignettes were clinically identical and differed only by patient age (35 or 65 years old); sex; race/ethnicity (white, Hispanic, or black); and socioeconomic status (occupation of lawyer or janitor). After viewing the vignette and indicating their management plans, physicians were also asked to discuss the challenges related to managing such a patient.

Results: Just over half (53\%) of physicians indicated that they would recommend a medication prescription for the vignette patient. Demographic characteristics (of the patient, physician, or practice) did not significantly influence this decision $(P>.1$ for all comparisons). Compared with physicians who did not recommend a diabetic-related prescription, physicians recommending therapy more often identified patient medication costs ( $74 \%$ vs $43 \%$ of physicians who would not increase therapy); medication adherence (63\% vs $49 \%$ ); and subsequent complications (34\% vs $22 \%)$ as important clinical issues in managing diabetes. Physicians not intensifying therapy more often indicated that they needed more clinical information (16\% vs $9 \%)$.

Conclusions: Using an experimental design we found that differences in the decision to intensify therapy were not significantly explained by patient, physician, or practice demographic variables. Physicians who intensified therapy were more likely to consider issues such as medication costs, patient adherence, and downstream complications. (J Am Board Fam Med 2009;22:513-520.)

Effective care of patients with type 2 diabetes generally requires the initiation and timely intensifica-

This article was externally peer reviewed.

Submitted 7 November 2008; revised 10 February 2009; accepted 13 February 2009.

From the Division of General Medicine, Massachusetts General Hospital and Harvard Medical School, Boston (RWG), and the New England Research Institute, Watertown (KEL, EG, CLL, LDM, JBM), MA.

Funding: Dr. Grant was supported by NIDDK Career Development Award (K23 DK067452). This study was supported by the National Institutes of Health, National Institute of Diabetes and Digestive and Kidney Diseases, DK66425.

Conflict of interest: none declared.

Corresponding author: Richard W. Grant, MD, MPH, 50-9 Staniford St, Boston, MA 02114 (E-mail: Rgrant@partners. org).

\footnotetext{
See Related Commentary on Page 471.
}

tion of medicines to control hyperglycemia, hypertension, and hyperlipidemia. ${ }^{1}$ Underscoring this requirement, the American Diabetes Association recently published expert guidelines that call for evaluation and corresponding medication adjustments on a 3 -month basis until glycemic control is achieved. ${ }^{2}$ Similarly, the Joint National Committee on Prevention, Detection, Evaluation, and Treatment of High Blood Pressure recommends monthly re-evaluation of medication efficacy for patients with above-goal hypertension. ${ }^{3}$ These guidelines are effectively applied in large clinical trials, where consenting patients (often prescreened to eliminate patients with poor adherence) are subject to aggressive protocols designed to achieve goals of control. ${ }^{4,5}$

In contrast, medication intensification in the usual care setting tends to follow a markedly slower pace. ${ }^{6}$ This slower rate of treatment intensification has been observed for all diabetes-related risk fac- 
tors and in multiple settings. ${ }^{7,8}$ One seminal study by Berlowitz et al, ${ }^{9}$ for example, found that, at a given clinic visit, hypertensive medications were adjusted in fewer than $10 \%$ of diabetic veterans with elevated blood pressure $(>160 / 90 \mathrm{~mm} \mathrm{Hg})$.

Studies of usual care practices have attributed the lack of medication intensification to a wide range of factors, including competing clinical demands during time-limited clinical encounters, lack of clinical guideline awareness, physician over-estimation of care provided, and the perceived likelihood of patient nonadherence. ${ }^{10-12}$ Attention has recently turned to developing interventions to increase the rate of medication intensification. ${ }^{13}$ However, because the key determinants of this phenomenon remain poorly understood, appropriate and effective targets for interventions are not known.

In the "real world" setting, patients tend to cluster by physician (eg, female patients with female physicians, minority patients with less experienced physicians) and within practice settings (eg, poorer patients in community health centers). ${ }^{14-16} \mathrm{Be}-$ cause of this clustering, observational studies of medication intensification may be confounded by the unmeasured variables that link patients to their own doctors. We used an experimental study design using a video case vignette to test the hypothesis that key patient (age, sex, race/ethnicity, socioeconomic status [SES]) and physician (experience, sex) demographic factors influenced the tendency to intensify medical therapy.

In addition to testing primary variables and interaction terms, we also used the video vignette viewing as the basis for conducting a qualitative analysis of physicians' explanations for their medication management recommendations. Our objective was to identify the major physician-reported barriers to and motivators of medication intensification.

\section{Methods}

\section{Study Design}

We conducted a vignette-based experiment to simultaneously measure the effects of patient attributes (age, sex, race/ethnicity, and SES) and physician characteristics (sex and years of clinical experience) on the decision to intensify therapy. In this factorial design, we tested each possible combination of the experimental variables (ie, patient attributes and physician characteristics). Study physicians viewed a 6-minute video vignette portraying a patient with type 2 diabetes. After viewing the entire vignette, participants completed survey questions about their evaluation and management decisions and then engaged in a structured interview about the challenges they would face in managing a patient like the one they just observed in the vignette. Study interviewers were carefully trained and certified, and selected tape-recorded interviews were reviewed by supervisors for quality control on a regular basis.

\section{Study Participants}

We enrolled 192 US primary care physicians active in clinical care and practicing in New Jersey, New York (excluding the New York City metropolitan area, Long Island, and the extreme northern counties), or Pennsylvania. Physicians were recruited from 2005 through 2007. Each study physician viewed one standardized clinical case vignette of a "patient" actor with type 2 diabetes and initial symptoms of foot neuropathy. To be eligible for selection, physicians had to (1) be internists or family practitioners; (2) have $\leq 12$ years of clinical experience (graduated between 1989 to 96 ) or $\geq 22$ years of clinical experience (graduated between 1965 to 79 ) to get clear separation by level of experience; (3) be currently in clinical practice more than half-time; and (4) be trained at an accredited medical school in the United States. We excluded international medical graduates because this study was part of a larger international project to compare physicians from different countries.

Screening telephone calls were conducted to identify eligible physiciana and a 1-hour appointment was scheduled for vignette viewing and a one-on-one, structured interview. Each physician was provided a modest stipend (\$100) to partially offset lost revenue and to acknowledge their participation. Institutional Review Board (Massachusetts General Hospital) approval was obtained for the study, and signed informed consents were collected from each participating physician.

\section{Development of Case Vignettes}

The script for the vignette was developed from several tape-recorded role-playing sessions with experienced clinical advisors (including a physician coinvestigator active in clinical practice; this physician was directly involved in script development 
Table 1. Symptoms, Patient Behavior, and Clinical Data Embedded in the Diabetes Vignette "Diagnosed Diabetes with Complications"

\begin{tabular}{lll}
\hline Patient Symptoms & Patient Behaviors & Clinical Data \\
\hline - Burning sensation at bottoms of feet & $\bullet$ Regularly tests glucose & $\bullet$ Patient is overweight \\
and up one ankle & & \\
- Foot pain is intermittent and hard & $\bullet$ Concerned about high blood pressure & $\bullet$ Last hemoglobin A1c level was 6.9\% \\
to localize & & \\
- Patient otherwise feeling "quite & $\begin{array}{l}\text { - Adherence to blood pressure medicine } \\
\text { good" }\end{array}$ & $\bullet$ Single high blood pressure reading (145/98) \\
\hline
\end{tabular}

from initial stages of role playing to latter stages of filming to maximize clinical authenticity). Professional actors were trained (under experienced physician supervision) to realistically portray a patient with type 2 diabetes in need of continuing evaluation and management. To better draw out between-physician differences, the vignette patient had "borderline" results: their last measured hemoglobin A1c value was $6.9 \%$ but the patient had symptoms consistent with early peripheral neuropathy, and the patient was concerned about his/her currently elevated blood pressure result (145/98 $\mathrm{mm} \mathrm{Hg}$ ) although previous readings had not been elevated. See Table 1 for a summary of the key clinical details embedded in the vignette.

The case vignettes differed only by patient age (35 or 65 years old), sex, race/ethnicity (white, black, or Hispanic), and SES (lower vs higher status, depicted by current or former employment as a janitor or a lawyer, respectively). A full factorial of $2^{3} \times 3=24$ combinations of patient age, sex, race/ethnicity, and SES was used for the video scenarios. One of the 24 combinations was shown to each physician (the 192 physicians were evenly divided by gender and experience). Previous studies have used similar methods with success. ${ }^{17-19}$

We took 3 steps to bolster the external validity of the case vignette. (1) We devoted considerable effort to ensuring the clinical authenticity of the videotaped presentation by basing the scripts on clinical experience, filming with experienced clinicians present, and by using professional actors/ actresses. (2) We presented the vignettes to study physicians in the context of their usual practice setting (not at a professional meeting, a course update, or in their home) so that it was likely they encountered real patients before and after they viewed the patient in the videotape. (3) We emphasized to participants at the outset that they should view the patient as one of their own patients and to respond as they would typically respond in their own practice. Most study physicians $(85 \%)$ considered the vignettes either very or reasonably typical of patients in their practice.

\section{Definition of Primary Outcome}

Study physicians were instructed to provide their responses based on the available clinical information presented in the vignette. Our primary outcome of interest was the physician's decision to intensify pharmacotherapy. After viewing the vignette, physicians were specifically asked, "What medication would you prescribe or recommend today?" We focused specifically on medications for glycemic, blood pressure, or lipid control and also created an aggregate outcome based on whether the study physician would prescribe medications for any one of these 3 diabetes-related risk factors.

\section{Practice Variables}

Although not part of the experimental factorial design, we collected information about the study physicians' practice environment. Response categories included practice size (solo, small group practice [2-10], or large group practice [ $>10$ physicians]); practice financial type (for-profit vs nonprofit); availability and use of clinical guidelines; and practice ownership (physician-owned or hospital-owned vs other).

\section{Qualitative Analyses}

After viewing the vignette, physicians were asked how they would treat the patient in terms of asking for additional information, performing physical examinations, ordering tests, prescribing medications, giving lifestyle advice, and referring to other physicians. These open-ended, structured interviews were designed to explore how participants made treatment decisions and what challenges/opportunities they faced with patients like the one 
seen in the vignette. Interviews were transcribed in full (19 audiotapes were excluded from analyses because of technical problems; $n=173$ transcripts).

Interview responses were coded using a modification of Strauss and Corbin's 3-step approach (open coding, axial coding, and selective coding). ${ }^{20}$ In the initial open coding phase, a sample of $15 \%$ of transcripts was reviewed line-by-line by 2 investigators (RWG and KEL) to identify relevant comments where physicians discussed medication prescription decisions. Using these guidelines, a research assistant identified all such comments in the overall dataset. A second $15 \%$ patient sample with relevant comments was then reviewed to identify core concepts related specifically to intensifying pharmacotherapy and to develop a detailed coding scheme that was applied to all excerpts where physicians discussed medication prescription decisions. Coders were blind to all patient and physician experimental factor assignments during this process. Coding differences were resolved by negotiation. The final codebook included 13 codes with detailed inclusion and exclusion criteria, examples of text segments, and other notes to assist the coding team. The qualitative analysis software package Atlas.ti (Atlas.ti Software Development GmBH, Berlin, Germany), was used to assist with the coding and management of the data.

\section{Statistical Methods}

For the quantitative analysis, we examined the role of patient, physician, and practice-level factors on the outcome of medication initiation. We used analysis of variance to test the main effects and 2-way interactions of the design variables (patient sex, race/ethnicity, age, and SES; physician sex and level of experience) on the decision to prescribe medicines on the day of the clinical encounter portrayed in the vignette. The balanced factorial design allowed for the unconfounded estimation of all main effects and 2-way interactions using analysis of variance. We used an availability sample to equally fill 4 design cells (sex by level of experience). The study was powered to find a $21 \%$ absolute difference between 2 groups (ie, 20\% vs 41\%) and $26 \%$ absolute difference between 3 groups (ie, $20 \%$ vs $46 \%$ ) at $80 \%$ power. Because the experiment was replicated, a pure error term with 96 degrees of freedom was used to test all effects using analysis of variance. SAS software (version 9, SAS
Institute, Inc., Cary, North Carolina) was used for all quantitative analyses.

\section{Results}

\section{Patient, Physician, and Practice Determinants of Medication Intensification}

After viewing the vignettes, $53 \%$ of study physicians indicated that they would recommend a diabetes-related medication prescription: $49 \%$ for antihypertensive therapy, $25 \%$ for antiglycemic therapy, and $15 \%$ for lipid-lowering therapy.

Differences in the decision to intensify therapy (with any of the 3 agents or for the aggregate measure of intensification) based on patient, physician, or practice factors did not reach statistical significance. However, we did note some trends. We found a $9 \%$ difference in medication intensification by patient age; $57 \%$ of physicians who viewed the older patient vignette recommended medications versus $48 \%$ of physicians who viewed the younger patient vignette. In addition, patients with lower SES tended to be intensified less often for each medication class (Table 2). Overall differences by patient race/ethnicity for the aggregate outcome were minimal; study physicians would intensify therapy for $52 \%$ of black, $52 \%$ of Hispanic, and $55 \%$ of white vignette patients. However, medication intensification for glycemic and lipid control tended to be less common among Hispanic vignette cases. Study physician responses to the case vignette did not differ significantly by the characteristics of the setting in which they practiced. Overall, $P>.05$ for all main effect comparisons and all but 2 of the 2-way interactions between patient, physician, and practice variables and the intensification outcome.

\section{Barriers to and Motivators of Treatment Intensification}

Physicians identified 3 major barriers to medication intensification: (1) $60 \%$ of physicians indicated concerns about medication costs for the patient (eg, resulting from insurer restrictions or formulary requirements); (2) 57\% considered the impact of patient medication adherence (eg, caused by increased regimen complexity or to lack of patient motivation); and (3) 13\% reported reluctance to make changes without more clinical data (eg, repeated blood pressure measurements). The major motivator of medication intensification was the desire to treat risk 
Table 2. Associations Between Patient, Physician, and Practice Characteristics and the Study Physician's Decision to Intensify Therapy to Treat Blood Pressure, Glycemia, Lipids, or Any of These Three Conditions After Viewing the Patient Vignette $(\mathbf{n}=192) *$

\begin{tabular}{|c|c|c|c|c|c|c|c|}
\hline \multirow[b]{2}{*}{ Medication Changes } & \multicolumn{7}{|c|}{ Intensified According to Vignette Patient Characteristics (\%) } \\
\hline & Male & Female & $P$ & & old & 65 years old & $P$ \\
\hline Blood pressure & 52 & 46 & .44 & \multicolumn{2}{|c|}{44} & 54 & .20 \\
\hline Glycemia & 24 & 25 & .88 & \multicolumn{2}{|c|}{22} & 27 & .46 \\
\hline Lipids & 18 & 13 & .32 & \multicolumn{2}{|c|}{14} & 17 & .55 \\
\hline \multirow[t]{2}{*}{ Any of the 3} & 55 & 50 & .52 & \multicolumn{2}{|c|}{48} & 57 & .24 \\
\hline & $\begin{array}{l}\text { Higher } \\
\text { SES }^{\dagger}\end{array}$ & $\begin{array}{l}\text { Lower } \\
\text { SES }^{\dagger}\end{array}$ & $P$ & \multicolumn{2}{|l|}{ White } & Hispanic & $P$ \\
\hline Blood pressure & 51 & 47 & .61 & 48 & 52 & 47 & .89 \\
\hline Glycemia & 29 & 20 & .18 & 28 & 27 & 19 & .51 \\
\hline Lipids & 20 & 10 & .08 & 17 & 17 & 11 & .53 \\
\hline \multirow[t]{3}{*}{ Any of 3} & 55 & 50 & .52 & 55 & 52 & 52 & .94 \\
\hline & \multicolumn{7}{|c|}{ Intensified According to Physician Characteristics $(\%)^{\ddagger}$} \\
\hline & Male & Female & $P$ & \multicolumn{2}{|c|}{ Less Experienced } & $\begin{array}{c}\text { More } \\
\text { Experienced }\end{array}$ & $P$ \\
\hline Blood pressure & 46 & 52 & .44 & \multicolumn{2}{|c|}{51} & 47 & .61 \\
\hline Glycemia & 23 & 26 & .66 & \multicolumn{2}{|c|}{26} & 23 & .66 \\
\hline Lipids & 14 & 17 & .55 & \multicolumn{2}{|c|}{13} & 18 & .32 \\
\hline \multirow[t]{3}{*}{ Any of 3} & 49 & 56 & .36 & & & 53 & .90 \\
\hline & \multicolumn{7}{|c|}{ Intensified According to Practice Factors $(\%)^{\S}$} \\
\hline & $\begin{array}{l}\text { For profit } \\
(\mathrm{n}=135)\end{array}$ & $\begin{array}{l}\text { Other } \\
(\mathrm{n}=57)\end{array}$ & $P$ & $\begin{array}{c}\text { Solo } \\
(\mathrm{n}=65)\end{array}$ & $\begin{array}{c}\text { Small } \\
(\mathrm{n}=105)\end{array}$ & $\begin{array}{c}\text { Large } \\
(\mathrm{n}=22)\end{array}$ & $P$ \\
\hline Blood pressure & 47 & 53 & .42 & 45 & 52 & 46 & .58 \\
\hline Glycemia & 24 & 25 & .68 & 25 & 27 & 14 & .39 \\
\hline Lipids & 14 & 18 & .15 & 20 & 12 & 14 & .68 \\
\hline Any of 3 & 50 & 58 & .30 & 49 & 56 & 46 & .47 \\
\hline
\end{tabular}

*Numbers are proportions of respondents indicating that they would prescribe medications for each or any of the three diabetesrelated risk factors; Numbers of physicians per practice variables are indicated in the table. Due to the factorial experimental design, numbers within each patient and physician variable category are equal.

${ }^{\dagger}$ Socioeconomic status (SES) depicted by occupation of lawyer versus janitor.

${ }^{\ddagger}$ Physician experience groups divided as $\leq 12$ years vs $\geq 22$ years of clinical experience.

${ }^{\$}$ Practice sizes defined by number of physicians $(1, \leq 10$, or $>10)$.

factors to avoid subsequent diabetes-related complications (mentioned by $28 \%$ of respondents).

Physicians who indicated that they would intensify therapy were also more likely to consider specific barriers and motivators when discussing the vignette in the subsequent qualitative interviews. Compared with physicians who would not intensify treatment for the vignette patient $(\mathrm{n}=81 ; 47 \%$ of respondents), physicians who would intensify treatment $(\mathrm{n}=92 ; 53 \%)$ more often considered medication costs $(74 \%$ vs $43 \%)$, patient medication adherence ( $63 \%$ vs $49 \%$ ), and subsequent diabetesrelated complications ( $34 \%$ vs $22 \%$ ) when making their clinical decisions. The only factor considered more often by the physicians who would not intensify therapy was the "need for more clinical information," noted by $16 \%$ of nonintensifiers and by $9 \%$ of intensifiers. Table 3 presents the treatment considerations reported by study physicians, contrasting physicians who indicated they would versus those that would not increase therapy.

In exploratory analyses we found that study physicians viewing vignettes portraying younger patients were more likely to report concerns about needing more clinical data (19\% vs $7 \%$ ). Physicians viewing vignettes portrayed by black actors may also have been more concerned about patient adherence $(66 \%$ vs $59 \%$ for white patient and $45 \%$ 
Table 3. Differences in Medication-Related Treatment Concerns Between Physicians Who Would or Would Not Prescribe Therapy for Vignette $(n=173) *$

\begin{tabular}{|c|c|c|c|c|c|c|}
\hline \multirow[b]{3}{*}{ Treatment Concerns (\%) } & \multicolumn{6}{|c|}{ Would Study Physician Prescribe a Medicine for Vignette Patient to Treat: } \\
\hline & \multicolumn{2}{|c|}{ Glycemia? } & \multicolumn{2}{|c|}{ Blood Pressure? } & \multicolumn{2}{|c|}{$\begin{array}{c}\text { Anything Related to } \\
\text { Diabetes? }\end{array}$} \\
\hline & $\begin{array}{c}\text { Yes } \\
(\mathrm{n}=45)\end{array}$ & $\begin{array}{c}\text { No } \\
(\mathrm{n}=128)\end{array}$ & $\begin{array}{c}\text { Yes } \\
(\mathrm{n}=85)\end{array}$ & $\begin{array}{c}\text { No } \\
(\mathrm{n}=88)\end{array}$ & $\begin{array}{c}\text { Yes } \\
(\mathrm{n}=92)\end{array}$ & $\begin{array}{c}\text { No } \\
(\mathrm{n}=81)\end{array}$ \\
\hline Financial barriers & $89^{\dagger}$ & 49 & $75^{+}$ & 44 & $74^{\dagger}$ & 43 \\
\hline Medication adherence & $71^{\dagger}$ & 52 & $65^{\dagger}$ & 49 & 63 & 49 \\
\hline Need more clinical data & 11 & 14 & 8 & 18 & 10 & 17 \\
\hline Long-term risk for complications & 38 & 25 & $37^{+}$ & 20 & 34 & 22 \\
\hline
\end{tabular}

*Numbers are proportions of study physicians who discussed each of the 4 treatment concerns, categorized by whether the study physician would prescribe medications for the indicated diabetes-related conditions. "Anything related to diabetes" defined as medicines to treat hypertension, hyperglycemia, or hyperlipidemia.

${ }^{\dagger}$ Difference between yes and no responses statistically significant $(P<.05)$.

for Hispanic patient vignettes) even though all vignette "patients" reported less than ideal adherence. Tests of 2-way interactions revealed that less experienced physicians were more concerned with adherence for patients with lower SES whereas more experienced physicians had greater adherence concerns among patients with higher SES $(P=$ .006). No other associations between reported barriers and patient or physician characteristics approached statistical significance.

\section{Discussion}

Medication prescribing and, in particular, the decision to intensify therapy for patients not meeting the goals of care represents 2 of the core physician tasks for the management of patients with type 2 diabetes. In thisarticle we provide new insight into the complex issue of medication intensification as it applies to a primary care patient with borderline indication for therapy change. We applied an innovative experimental design to investigate the factors that influence physicians' medical decision making. By exposing study participants to clinically identical case presentations, our factorial experiment permitted an assessment of clinical decision making unconfounded by the clustering of patient and physician characteristics seen in real world settings. We found that experimentally varying patient demographic variables while preserving the identical clinical content in the vignettes did not uncover major influences of patient race, sex, SES, or age on the physician's decision to make medication recommendations. However, we cannot exclude smaller (though clinically significant) effects of clinical decision making on health care disparities.

Our study extends prior work by Schulman et $\mathrm{al}^{21}$ who used actors to portray a chest pain scenario. In the Schulman study, physician estimates of coronary artery disease probability were $5 \%$ lower in women than men and 6\% lower in 55 year-old versus 70-year-old vignette patients. Moreover, physicians were significantly less likely to refer women or black patients for cardiac catheterization. We found similar magnitude (although not statistically significant) differences by patient demographic characteristics, raising the possibility that the decision to intensify therapy in the primary care setting may be influenced by a similar mechanism as the more acute decision to refer for catheterization. When we explored 2-way interactions we found that less experienced physicians were more concerned with adherence for patients with lower SES, whereas more experienced physicians had greater adherence concerns among patients with higher SES. This result suggests that SESrelated differences may be mediated by physician experience.

Our study revealed a wide range of physician practice styles in response to the "borderline" case presented. This practice variation implies that physicians weigh additional, unmeasured considerations when making treatment decisions. In previous work by our group, we found that physicians used patients' demographic characteristics only as a starting point in their assessments, and they proceeded to make detailed assessments about cognitive ability, motivation, social support, and other 
factors they considered predictive of adherence with medical recommendations and therefore relevant to treatment decisions. ${ }^{22}$ This current study extends our previous work by highlighting the primary factors (cost, presumed adherence, extent of available data, and potential for long-term complications) that are considered during the medication intensification decision-making process.

By contrasting physicians who would or would not recommend therapy for the vignette patient, the medication intensification barriers physicians identified in this study provide some insight into the nonclinical factors that drive decision making. We found that study physicians were primarily concerned with 3 barriers when deciding whether to intensify therapy: (1) medication costs to the patient, (2) likely medication adherence, and (3) the need for additional clinical data before making a decision (particularly among younger patients). Of interest, physicians who chose to intensify therapy also tended to consider more potential patient barriers to taking medication. We hypothesize that this may be a reflection of a more empathic practice style. Future research will be needed to ascertain whether a patient-centered care practice style is associated with more frequent medication intensification.

The results of our study must be interpreted in the context of the study design. Our primary outcome of medication intensification represents only a single component of diabetes care and thus does not address other domains such as counseling, referrals, and clinical integration of new information. The strength of our approach (eg, use of identical patient presentations rather than physicians' own patients) by necessity also deprives our study participants of the detailed knowledge they may have of their own patients. Thus, clinical decisions in the experimental setting may not fully represent usual care practices. However, prior research studies comparing vignettes with standardized patients and other methods corroborate the result that vignettes are ecologically valid for studies of medical decision making. ${ }^{17-19}$ Finally, our study was designed to detect group-level biases among primary care physicians as a class. This study was therefore unable to ascertain whether some physicians have gender, age, or race biases but others do not.

\section{Conclusion}

We used a combination of experimental, quantitative, and qualitative methods to examine the impact of patient, physician, and practice characteristics in the management of patients with type 2 diabetes. Using an otherwise identical clinical case vignette, we were unable to identify major demographic predictors of medication intensification. However, we found that physicians who intensified therapy were also more likely to consider specific barriers to medications use.

\section{References}

1. American Diabetes Association. Standards of medical care in diabetes-2008. Diabetes Care 2008; 31(Suppl 1):S12-S54.

2. Nathan DM, Buse JB, Davidson MB, et al. Management of hyperglycemia in type 2 diabetes: a consensus algorithm for the initiation and adjustment of therapy: a consensus statement from the American Diabetes Association and the European Association for the Study of Diabetes. Diabetes Care 2006;29: 1963-72.

3. Chobanian AV, Bakris GL, Black HR, et al. The Seventh Report of the Joint National Committee on Prevention. Detection, Evaluation, and Treatment of High Blood Pressure. JAMA 2003;289:2560-72.

4. Intensive blood-glucose control with sulphonylureas or insulin compared with conventional treatment and risk of complications in patients with type 2 diabetes (UKPDS 33). UK Prospective Diabetes Study (UKPDS) Group. Lancet 1998;352:837-53.

5. Yusuf S, Sleight P, Pogue J, Bosch J, Davies R, Dagenais G. Effects of an angiotensin-convertingenzyme inhibitor, ramipril, on cardiovascular events in high-risk patients. The Heart Outcomes Prevention Evaluation Study Investigators. N Engl J Med 2000;342:145-53.

6. Grant RW, Buse JB, Meigs JB. Quality of diabetes care in US academic medical centers: low rates of medical regimen change. Diabetes Care 2005;28: 337-442.

7. Shah BR, Hux JE, Laupacis A, Zinman B, van Walraven $C$. Clinical inertia in response to inadequate glycemic control: do specialists differ from primary care physicians? Diabetes Care 2005;28:600-6.

8. Rodondi N, Peng T, Karter AJ, et al. Therapy modifications in response to poorly controlled hypertension, dyslipidemia, and diabetes mellitus. Ann Intern Med 2006;144:475-84.

9. Berlowitz DR, Glickman M, Ash AS, Friedman R, Hickey EC, Kader B. Hypertension management in patients with diabetes. Diabetes Care 2003;26:355-9.

10. Safford MM, Shewchuk R, Qu H, et al. Reasons for not intensifying medications: differentiating "clinical inertia" from appropriate care. J Gen Intern Med 2007;22:1648-55.

11. Parchman ML, Pugh JA, Romero RL, Bowers KW. Competing demands or clinical inertia: the case of 
elevated glycosylated hemoglobin. Ann Fam Med 2007;5:196-201.

12. Grant RW, Adams AS, Trinacty CM, et al. Relationship between patient medication adherence and subsequent clinical inertia in type 2 diabetes glycemic management. Diabetes Care 2007;30:807-12.

13. Perlin JB, Pogach LM. Improving the outcomes of metabolic conditions: managing momentum to overcome clinical inertia. Ann Intern Med 2006;144: 525-7.

14. Fang MC, McCarthy EP, Singer DE. Are patients more likely to see physicians of the same sex? Recent national trends in primary care medicine. Am J Med 2004;117:575-81.

15. Bach PB, Pham HH, Schrag D, Tate RC, Hargraves JL. Primary care physicians who treat blacks and whites. N Engl J Med 2004;351:575-84.

16. Hasnain-Wynia R, Baker DW, Nerenz D, et al. Disparities in health care are driven by where minority patients seek care. Arch Intern Med 2007;167: 1233-88.

17. Feldman HA, McKinlay JB, Potter DA, et al. Nonmedical influences on medical decision making: an experimental technique using videotapes, factorial design, and survey sampling. Health Serv Res 1997; 32:343-66.

18. Peabody JW, Luck J, Glassman P, Dresselhaus TR, Lee M. Comparison of vignettes, standardized patients, and chart abstraction: a prospective validation study of 3 methods for measuring quality. JAMA 2000;283:1715-22.

19. McKinlay JB, Lin T, Freund K, Moskowitz M. The unexpected influence of physician attributes on clinical decisions: results of an experiment. J Health Soc Behav 2002;43:92-106.

20. Strauss A, Corbin J. Basics of qualitative research: grounded theory procedures and techniques. Newbury Park, CA: Sage; 1990.

21. Schulman KA, Berlin JA, Harless W, et al. Correction: The effect of race and sex on physicians' recommendations for cardiac catheterization. N Engl J Med 1999;340:618-26.

22. Lutfey KE, Campbell SM, Renfrew MR, Marceau LD, Roland M, McKinlay JB. How are patient characteristics relevant for physicians' clinical decision making in diabetes? An analysis of qualitative results from a cross-national factorial experiment. Soc Sci Med 2008;67:1391-9. 